STOCHASTIC MODELING AND CONTROL

BANACH CENTER PUBLICATIONS, VOLUME 122

INSTITUTE OF MATHEMATICS

POLISH ACADEMY OF SCIENCES

WARSZAWA 2020

\title{
BELLMAN EQUATIONS FOR SCALAR LINEAR CONVEX STOCHASTIC CONTROL PROBLEMS
}

\author{
TYRONE DUNCAN and BOZENNA PASIK DUNCAN \\ Department of Mathematics, University of Kansas \\ Lawrence, KS 66045 \\ ORCID: 0000-0002-6664-9967, 0000-0002-3316-6475 \\ E-mail: duncan@ku.edu,bozenna@ku.edu \\ ŁUKASZ STETTNER \\ Institute of Mathematics, Polish Academy of Sciences \\ Śniadeckich 8, 00-656 Warszawa, Poland \\ ORCID:0000-0002-7441-9002_E-mail: stettner@impan.pl
}

\begin{abstract}
A family of discrete time stochastic control problems with linear dynamics and convex cost functionals are studied. For the case of a scalar control for such a model with additive finite time horizon, discounted, and average cost per unit time convex cost functionals as well as multiplicative (exponential) finite time horizon, discounted and long run average convex functionals explicit solutions are described for suitable Bellman equations. In the particular case of a linear quadratic control problem a general continuous time problem is described. The form of the optimal strategies for each of these control problems is characterized.
\end{abstract}

1. Introduction. In this paper the primary model for control is a one dimensional discrete time stochastic dynamical system described as follows on given probability space $(\Omega, \mathcal{F}, \mathcal{P})$ :

$$
x_{n+1}=a_{n} x_{n}+b_{n} u_{n}+c_{n} W_{n+1},
$$

where $n=0,1, \ldots, T-1, a_{n}, b_{n}$ and $c_{n}$ are constants, $\left(W_{n}\right)$ is a sequence of independent and identically distributed (i.i.d.) symmetric random variables. Various cost functionals

2010 Mathematics Subject Classification: Primary: 93E20; Secondary: 60J20, 93E24.

Key words and phrases: convex cost functionals; linear stochastic discrete time dynamics; scalar control problems, finite; discounted and long run cost functional.

The paper is in final form and no version of it will be published elsewhere. 
are considered consisting of strictly convex, even functions that are additive or exponential with finite time horizons or infinite time horizons either discounted or long run average. The results given here can be generalized for finite time horizon problems to multidimensional control models. Finite time horizon linear convex problems with linear cost functions with respect to control were studied in discrete time in [2] using a deterministic approach, and then generalized to continuous time in [13. For infinite time horizon problems some special properties are required that are typical for scalar controls. The assumption concerning independence of the sequence of noise in the case of finite horizon problems can be relaxed by assuming only symmetry of the conditional laws (which justifies and extends the results of the papers [7] and [8]). The case with finite time horizon can also be studied in continuous time for a general noise and for quadratic functionals using the family of nearly optimal controls consisting of linear controls of the current state of the process. The purpose of this paper is to show that linear stochastic dynamics and the functions in the cost functionals being strictly convex allows one to find solutions to suitable Bellman equations for various kinds of stochastic control problems. While the explicit forms of optimal controls are not obtained as occurs in the case of linear quadratic problems, there are inductive characterizations for optimal controls. This work generalizes several papers in various directions (see [6], [5] and references therein). An infinite horizon discrete time linear convex problem was considered in [1]. A finite horizon discrete time linear convex Gaussian problem with partial observation is considered e.g. in [10]. Continuous linear convex problems were also studied using a stochastic maximum principle in [4. In the first part of this paper additive functionals consisting of strictly convex, even functions are studied and optimal controls for finite horizon problems, discounted and average cost per unit time problems are characterized. In the second part of the paper exponential (multiplicative) functionals are considered and solved for finite horizon, discounted and long run average control problems. The main novelty of the paper consists in a unified approach to discrete time linear convex control problems, allowing in certain cases to consider general noise processes and to find the particular form of optimal controls for general discrete and continuous time linear quadratic control problems. The results concerning infinite time horizon linear convex problems seem to be original in particular a solution to an average cost per unit time control problem for a discrete time linear convex problem. Furthermore solutions to the Bellman equations corresponding to long run exponential functionals are obtained. This latter result is obtained in a weaker form as a limit of finite horizon exponential problems and in a stronger form using a vanishing discount approach.

2. Finite horizon problem. For the model with dynamics (1) in this section the following finite horizon additive functional is considered

$$
J_{x}^{T}(V)=E_{x}\left[\sum_{t=0}^{T-1}\left(f_{t}\left(x_{t}\right)+g_{t}\left(u_{t}\right)\right)+G\left(x_{T}\right)\right]
$$

which is minimized over all strategies $V=\left(u_{0}, \ldots, u_{T-1}\right)$, that are adapted, that is, $u_{t}$ is measurable with respect to the filtration $\mathcal{F}_{t}=\sigma\left\{W_{n}, n \leq t\right\}$ for $t=1,2, \ldots$ It is also noted that the probability measure $\mathcal{P}_{x}$, and therefore its expected value is conditional 
given the initial point $x_{0}=x$ of the model (1). The right hand side of $(2)$ depends on $V$ since dynamics of $\left(x_{t}\right)$ is given by (1). Therefore we could write $\left(x_{t}^{V}\right)$ but we shall not point out this dependence whenever it would not lead to ambiguity. It is assumed that the functions $f_{t}, g_{t}$ and $G$ are even and strictly convex. It is clear that then their minima occur at 0 . The following lemmas are used subsequently.

LEMMA 2.1. When $F$ is strictly convex (resp. convex) and even then, assuming integrability of $F\left(z+c W_{1}\right)$ for a constant $c$, we infer that the function $\tilde{F}$ given by

$$
\tilde{F}(z)=E\left[F\left(z+c W_{1}\right)\right]
$$

is also even and strictly convex (resp. convex).

Proof. Strict convexity of $\tilde{F}$ follows from strict convexity of $F$. $\tilde{F}$ is even because $F$ is even and the random variable $W_{1}$ is symmetric. The case for convex $F$ follows analogously.

LEMMA 2.2. For strictly convex (resp. convex) and even functions $\tilde{F}$ and $g$ and constants $a$ and $b$ the following function

$$
H(x)=\inf _{u}[g(u)+\tilde{F}(a x+b u)]
$$

is even and strictly convex (resp. convex). Furthermore its minimum is uniquely attained in the interval $\left[0, \frac{-a x}{b}\right]$ if $\frac{-a x}{b}>0$ and in the interval $\left[\frac{-a x}{b}, 0\right]$ if $\frac{-a x}{b}<0$, and at 0 if $a=0$. Note that $H(0)=g(0)+\tilde{F}(0)$ that is for $x=0$ the optimal control is $u=0$.

Proof. It is clear that the infimum (4) is attained. For arbitrary $x, x^{\prime}$ there are $u, u^{\prime}$ such that $H(x)=[g(u)+\tilde{F}(a x+b u)]$ and $H\left(x^{\prime}\right)=\left[g\left(u^{\prime}\right)+\tilde{F}\left(a x^{\prime}+b u^{\prime}\right)\right]$. Then for $\lambda \in(0,1)$ taking into account strict concavity of $\tilde{F}$ and $g$ we have

$$
\begin{aligned}
& H\left(\lambda x+(1-\lambda) x^{\prime}\right) \leq g\left(\lambda u+(1-\lambda) u^{\prime}\right)+\tilde{F}\left(\lambda(a x+b u)+(1-\lambda)\left(a x^{\prime}+b u^{\prime}\right)\right) \\
& <\lambda(g(u)+\tilde{F}(a x+b u))+(1-\lambda)\left(g\left(u^{\prime}\right)+\tilde{F}\left(a x^{\prime}+b u^{\prime}\right)\right)=\lambda H(x)+(1-\lambda) H\left(x^{\prime}\right)
\end{aligned}
$$

so that $H$ is strictly convex. Now

$$
\begin{aligned}
H(-x)=\inf _{u}[g(u)+\tilde{F} & (-a x+b u)] \\
& =\inf _{u^{\prime}}\left[g\left(-u^{\prime}\right)+\tilde{F}\left(-a x-b u^{\prime}\right)\right]=\inf _{u^{\prime}}\left[g\left(u^{\prime}\right)+\tilde{F}\left(a x+b u^{\prime}\right)\right]
\end{aligned}
$$

using the fact that $g$ and $\tilde{F}$ are even. Therefore $H$ is even as well. The case when $\tilde{F}$ and $g$ are only convex can be shown almost identically. The function $H$ is an infimum of the sum of two convex in $u$ functions $g(u)$ and $\tilde{F}(a x+b u)$. They attain their infima at $u=0$ and $u=\frac{-a x}{b}$ respectively. Therefore the infimum of the sum is somewhere between $u=0$ and $u=\frac{-a x}{b}$, and by strict convexity there is a unique point where it is attained.

Consequently the following theorem can be verified.

THEOREM 2.3. Assume that the functions $f_{t}, g_{t}$ and $G$ are nonnegative, strictly convex and even and for each $x$

$$
E_{x}\left[\sum_{t=0}^{T-1-k} f_{t}\left(x_{t}\right)+G\left(x_{T-k}\right)\right]<\infty
$$


for $k=0,1, \ldots, T-1$, where $\left(x_{n}\right)$ corresponds to the state process satisfying (1) with control $u \equiv 0$. Then there is a sequence of strictly convex even functions $w_{t}, t=0,1, \ldots, T$, that are solutions to the following system of Bellman equations:

$$
\begin{aligned}
& w_{T}(x)=G(x) \\
& w_{n}(x)=f_{n}(x)+\inf _{u}\left[g_{n}(u)+E\left[w_{n+1}\left(a_{n} x+b_{n} u+c_{n} W_{n+1}\right)\right]\right], n=T-1, \ldots, 0 .
\end{aligned}
$$

Furthermore $w_{0}(x)=\inf _{V} J_{x}^{T}(V)$, and the optimal strategies are in the form $\hat{u}_{n}\left(x_{n}\right)$ at time $n$, where $\hat{u}_{n}$ are selectors to the equations (8), which are continuous functions.

Proof. By the assumption (7) it follows that equations (8) are well defined. By Lemmas 2.1 and 2.2 the functions $w_{n}$ are even and strictly convex. Since the selectors are defined in a unique way (by strict concavity) they are also continuous. The proof that $w_{0}(x)=$ $\inf _{V} J_{x}^{T}(V)$ follows in a standard way (see Section 3.2 of [11] or Theorem 4.1.1 of [15]).

Consider now the case of a general noise process. Assume that random variables $\left(W_{n}\right)$ are not independent but

$$
\text { conditional laws } P\left\{W_{n+1} \in \cdot \mid W_{1}, W_{2}, \ldots, W_{n}\right\} \text { are symmetric. }
$$

This assumption covers in particular the cases (in one dimension) given in the papers [7] and [8]. This law can be chosen as a regular conditional probability and define the system of Bellman equations

$$
\begin{aligned}
& w_{T}(x)=G(x) \\
& \begin{aligned}
w_{n}(x)=f_{n}(x)+\inf _{u}\left[g_{n}(u)+E\left[w_{n+1}\left(a_{n} x+b_{n} u+c_{n} W_{n+1}\right) \mid W_{1}, \ldots, W_{n}\right]\right], \\
n=T-1, T-2, \ldots, 1
\end{aligned} \\
& w_{0}(x)=f_{0}(x)+\inf _{u}\left[g_{0}(u)+E\left[w_{1}\left(a_{0} x+b_{0} u+c_{0} W_{1}\right)\right]\right] .
\end{aligned}
$$

Notice now that the functions $\left(w_{t}\right)$ defined above are random since they depend on conditional expectations. Conditional expectations considered as with respect to regular conditional probabilities are defined everywhere. Functions $\left(w_{t}\right)$ exist, i.e. are finite under suitable integrability conditions. The following theorem explains solvability of the system 9 .

THEOREM 2.4. Assume that the functions $f_{t}, g_{t}$ and $G$ are nonnegative, strictly convex and even and the right hand sides of $\left(9\right.$ are well defined. Then there is set $\Omega^{\prime}$ such that $P\left(\Omega^{\prime}\right)=1$ and a sequence of convex even random functions $w_{t}(\cdot, \omega), t=0,1, \ldots, T$, which for $\omega \in \Omega^{\prime}$ are solutions to the system of Bellman equations (9). Furthermore $w_{0}(x)=\inf _{V} J_{x}^{T}(V)$, and the optimal strategies are in the form $\hat{u}_{n}\left(x_{n}, \omega\right)$ at time $n$, where $\hat{u}_{n}$ are for $\omega \in \Omega^{\prime}$ selectors to the equations (9) and are continuous functions.

Proof. Since we consider regular conditional probabilities we can use Theorem 5 of $[9]$ to guarantee measurability of random functions $w_{t}(\cdot, \omega), t=0,1, \ldots, T$. Note that selectors to the equations (9) are random and by Theorem 5 of [9] measurable. In the system (9) we are allowed to replace essential infimum by infimum since selectors by strict convexity of $f_{t}, g_{t}$ and $G$ are uniquely defined and therefore are continuous functions. The last part, i.e. identity $w_{0}(x)=\inf _{V} J_{x}^{T}(V)$, follows in a similar way as in Section 3.2 of [11] or Theorem 4.1.1 of [15]. 
3. General stochastic linear quadratic problems. In this section initially quadratic functionals are considered, that is, the case where $f_{t}(x)=q_{t} x^{2}, g_{t}(u)=e_{t} u^{2}$ and $G(x)=l x^{2}$ with positive constants $q_{t}, e_{t}$ and $l$ are positive deterministic functions for discrete time dynamics (1), and the results of Theorem 2.4 are used.

COROLlary 3.1. Assume that functions $f_{t}, g_{t}$ and $G$ are quadratic (as above) and the conditional laws of the noise sequence $W_{n}$ are symmetric and their conditional second moments are finite. Then $w_{0}$ is a quadratic function and an explicit form for an optimal control is $\hat{u}_{n}\left(x_{n}, \omega\right)=k_{n} x_{n}$, where $k_{n}$ is deterministic.

Proof. A standard completion of squares method is used. It is only necessary to show that if $w_{n+1}(x)=r_{1} x^{2}+r_{2}$ for constant $r_{1}>0$, where $r_{2}$ is random, then $w_{n}$ determined from $(9)$ is also of the same form and an optimal value of $u$ occurs as in the statement of this corollary. Since $E\left[W_{n+1} \mid W_{1}, \ldots, W_{n}\right]=0$ it follows that

$$
\begin{aligned}
& w_{n}(x)= q_{n} x^{2}+\inf _{u}\left[e_{n} u^{2}+E\left[r_{1}\left(a_{n} x+b_{n} u+c_{n} W_{n+1}\right)^{2}+r_{2} \mid W_{1}, \ldots, W_{n}\right]\right] \\
&=q_{n} x^{2}+\inf _{u}\left[e_{n} u^{2}+r_{1}\left(a_{n}^{2} x^{2}+b_{n}^{2} u^{2}+c_{n}^{2} E\left[W_{n+1}^{2} \mid W_{1}, \ldots, W_{n}\right]\right.\right. \\
&\left.\left.+2 a_{n} x b_{n} u+2\left(a_{n} x+b_{n} u\right) c_{n} E\left[W_{n+1} \mid W_{1}, \ldots, W_{n}\right]\right)+E\left[r_{2} \mid W_{1}, \ldots, W_{n}\right]\right] \\
&=\left(q_{n}+r_{1} a_{n}^{2}\right) x^{2}+\inf _{u}\left[\left(e_{n}+r_{1} b_{n}^{2}\right) u^{2}+2 r_{1} a_{n} x b_{n} u\right] \\
& \quad+r_{1} c_{n}^{2} E\left[W_{n+1}^{2} \mid W_{1}, \ldots, W_{n}\right]+E\left[r_{2} \mid W_{1}, \ldots, W_{n}\right] \\
&\left.\quad+q_{n}+r_{1} a_{n}^{2}\right) x^{2}+\inf _{u}\left[\left(e_{n}+r_{1} b_{n}^{2}\right)\left(u+\frac{r_{1} a_{n} b_{n} x}{e_{n}+r_{1} b_{n}^{2}}\right)^{2}-\frac{r_{1}^{2} a_{n}^{2} b_{n}^{2} x^{2}}{e_{n}+r_{1} b_{n}^{2}}\right] \\
& \quad+r_{1} c_{n}^{2} E\left[W_{n+1}^{2} \mid W_{1}, \ldots, W_{n}\right]+E\left[r_{2} \mid W_{1}, \ldots, W_{n}\right] \\
&=\left(q_{n}+r_{1} a_{n}^{2}-\frac{r_{1}^{2} a_{n}^{2} b_{n}^{2}}{e_{n}+r_{1} b_{n}^{2}}\right) x^{2}+r_{1} c_{n}^{2} E\left[W_{n+1}^{2} \mid W_{1}, \ldots, W_{n}\right]+E\left[r_{2} \mid W_{1}, \ldots, W_{n}\right]
\end{aligned}
$$

where note that $q_{n}+r_{1} a_{n}^{2}-\frac{r_{1}^{2} a_{n}^{2} b_{n}^{2}}{e_{n}+r_{1} b_{n}^{2}}>0$, and an optimal $u$ is in the form $-\frac{r_{1} a_{n} b_{n} x}{e_{n}+r_{1} b_{n}^{2}}$. The remaining part of the proof follows by induction.

Now consider a continuous time linear equation

$$
\dot{x}_{t}=\left(a x_{t}+b u_{t}\right) d t+c d W_{t}
$$

with general noise $\left(W_{t}\right)$ with finite second moments, symmetric conditional increments, as $P\left\{W_{t+s}-W_{t} \mid W_{u}, u \leq t\right\}$ and continuous trajectories. It is assumed that the control process $\left(u_{t}\right)$ is dyadic, that is, it is piecewise constant, adapted to the filtration $\mathcal{F}_{t}=$ $\sigma\left\{W_{s}, s \leq t\right\}$ and changed only at the multiples of some $\Delta$. The solution to (11) is understood in the time interval $[0, \Delta]$ in the following way

$$
y(t)=x_{0}+b u_{0} t+c\left(W_{t}-W_{0}\right)
$$

for $t \in[0, \Delta]$. Clearly $y(t)$ is continuous and consider $z$ as solution to the equation

$$
\dot{z}(t)=a(z(t)+y(t))
$$

with $z(0)=0$. Then one can show that $x_{t}=z(t)+y(t)$ is the solution to 11 . Consequently we obtain

$$
x_{t}=e^{a t} x_{0}+b u_{0}\left(t+\left(1+\frac{1}{a}\right)\left(e^{a t}-1\right)\right)+a \int_{0}^{t} e^{a(t-s)} c\left(W_{s}-W_{0}\right) d s
$$


for $t \leq \Delta$. Consider now the quadratic cost functional

$$
J_{x}^{T, c}(V)=E_{x}\left[\int_{0}^{T}\left(f\left(x_{t}\right)+g\left(u_{t}\right)\right) d t+G\left(x_{T}\right)\right]
$$

with $f(x)=q x^{2}, g(u)=e u^{2}$ and $G(x)=l x^{2}$, where $V=\left(u_{t}\right)$. If we assume for simplicity that $T$ is a multiple of $\Delta$ and $\left(u_{t}\right)$ is fixed on intervals of the length $\Delta$ it follows that

$$
J_{x}^{T, c}(V)=E_{x}\left[\sum_{i=0}^{T / \Delta-1} \int_{i \Delta}^{(i+1) \Delta}\left(f\left(x_{t}\right)+g\left(u_{i \Delta}\right)\right) d t+G\left(x_{T}\right)\right] .
$$

Now consider the following sequence of random backward Bellman equations (compare to (9)):

$$
\begin{aligned}
& w_{T}(x)=G(x) \\
& w_{i \Delta}(x)=\inf _{u}\left[e u^{2} \Delta+E\left[\int_{0}^{\Delta} f\left(x_{s}\right) d s+w_{(i+1) \Delta}\left(d_{1} x+d_{2} u\right.\right.\right. \\
& \left.\left.\left.\quad+a \int_{0}^{\Delta} e^{a(\Delta-s)} c\left(W_{i \Delta+s}-W_{i \Delta}\right) d s\right) \mid F_{i \Delta}\right]\right], \quad i=T / \Delta-1, \ldots, 0,
\end{aligned}
$$

with $d_{1}=e^{a \Delta}, d_{2}=b\left(\Delta+\left(1+\frac{1}{a}\right)\left(e^{a \Delta}-1\right)\right)$, and $\left(x_{s}\right)$ appearing in the formula for $w_{i \Delta}$ being solution to (14) with $W_{s}-W_{0}$ replaced by $W_{i \Delta+s}-W_{i \Delta}$. The following proposition provides a solution to the family of Bellman equations.

Proposition 3.2. Assume that the right hand sides of (17) are well defined. Then there is $\Omega^{\prime}$, such that $P\left(\Omega^{\prime}\right)=1$ and a sequence of convex even random functions $w_{t}(\cdot, \omega)$, $t=0,1, \ldots, T$, which for $\omega \in \Omega^{\prime}$ are solutions to the system of Bellman equations (17). For each fixed $\Delta$ the optimal strategy is of the form $u_{n \Delta}=k_{n} x_{n \Delta}$ with deterministic $k_{n}$. Furthermore each solution to (17) is of the form $w_{i \delta}=r_{1, n} x^{2}+r_{2, n}$ where $r_{1, n}$ is deterministic while $r_{2, n}$ can be random.

Proof. Since the family of functions $w_{n \Delta}$ are random use [9] to have measurable versions of these functions. When $w_{T}(x)=l x^{2}$, it suffices to show the step from $w_{(n+1) \Delta}$ to $w_{n \Delta}$. Assume that $w_{(n+1) \Delta}(x)=r_{1, n+1} x^{2}+r_{2, n+1}$. Then under the infimum sign for $w_{n}(x)$ it follows (using the fact that $E\left[W_{i \Delta+s}-W_{i \Delta} \mid F_{i \Delta}\right]=0$ )

$$
\begin{aligned}
& e u^{2} \Delta+E\left[\int _ { 0 } ^ { \Delta } q \left(x e^{a s}+b u\left(s+\left(1+\frac{1}{a}\right)\left(e^{a s}-1\right)\right)\right.\right. \\
& \left.+a \int_{0}^{s} e^{a(s-r)} c\left(W_{r+n \Delta}-W_{n \Delta}\right) d r\right)^{2} d s+r_{1, n+1}\left(x e^{a s}+b u\left(s+\left(1+\frac{1}{a}\right)\left(e^{a s}-1\right)\right)\right. \\
& \left.\left.\quad+a \int_{0}^{s} e^{a(s-r)} c\left(W_{r+n \Delta}-W_{n \Delta}\right) d r\right)^{2}+r_{2, n+1} \mid F_{n \Delta}\right] \\
& =h_{1} x^{2}+h_{2} u^{2}+h_{3} x u+q_{4}=\left(h_{1}-\frac{h_{3}^{2}}{2 h_{2}}\right) x^{2}+h_{2}\left(u+\frac{h_{3}}{2 h_{2}} x\right)^{2}+h_{4}
\end{aligned}
$$

with $h_{1}>\frac{h_{3}^{2}}{2 h_{2}}$, where $h_{4}$ can be random, while $h_{1}, h_{2}$ and $h_{3}$ are deterministic. Therefore the optimal (minimal) $u$ is of the form $-\frac{h_{3}}{2 h_{2}} x$, which by induction completes the proof. 
REMARK 3.3. In the case of continuous time it is necessary to define the class of controls $\left(u_{s}\right)$ for which equation (14) is satisfied. Therefore the family of piecewise constant controls seems to be reasonable. When a solution in a certain sense is given to 14 then it is necessary to introduce some kind of Ito's formula and some Riccatti equation (see [5] or [7]). In the case here the main result states that whenever there is restriction to dyadic controls then nearly optimal controls are of the form $k_{t} x_{t}$ for dyadic $t$, where $k$ is deterministic. This result is valid for very general stochastic noise processes $\left(W_{t}\right)$ provided that their increments are conditionally symmetric and the right hand sides of equation (17) are well defined.

4. Discounted control problem. Now a discounted control problem with an i.i.d. noise sequence $\left(W_{n}\right)$ in the dynamics of the state equation in the following form is considered

$$
x_{n+1}=a x_{n}+b u_{n}+c W_{n+1}
$$

for $n=0,1, \ldots$, with $x_{0}=x$. It is desired to minimize the following cost functional for a given fixed $\beta \in(0,1)$

$$
J_{x}^{\beta}(V)=E_{x}\left[\sum_{t=0}^{\infty} \beta^{t}\left(f\left(x_{t}\right)+g\left(u_{t}\right)\right)\right] .
$$

A function $w^{\beta}$ is sought as a solution to

$$
w^{\beta}(x)=f(x)+\inf _{u}\left[g(u)+\beta E\left[w^{\beta}\left(a x+b u+c W_{1}\right)\right]\right] .
$$

For a function $v$ let

$$
T^{\beta} v(x):=f(x)+\inf _{u}\left[g(u)+\beta E\left[v\left(a x+b u+c W_{1}\right)\right]\right] .
$$

In what follows it is assumed that the functions $g$ and $f$ are nonnegative, strictly convex and even.

THEOREM 4.1. Assume that $m_{\beta}(x):=E\left[\sum_{i=0}^{\infty} \beta^{i} f\left(x_{i}\right)\right]<\infty$ where $\left(x_{i}\right)$ corresponds to the state process with control $u_{n} \equiv 0$, for $n=0,1, \ldots$ Then there is a solution $w$ to the Bellman equation (21), which is convex, even and $w^{\beta}(x)=\inf _{V} J_{x}^{\beta}(V)$.

Proof. Let $w^{\beta, n}(x)=\left(T^{\beta}\right)^{n} 0(x)$. Since $f$ and $g$ are nonnegative $w^{\beta, n+1}(x) \geq w^{\beta, n}(x)$. Moreover by Lemmas 2.1 and 2.2 the functions $w^{\beta, n}$ are strictly convex and even. Since as one can show $\left(T^{\beta}\right)^{n} 0(x)$ (see e.g. Theorem 4.1.1. of [15]) is an optimal value of the functional

$$
J_{x}^{\beta, n}(V)=E_{x}\left[\sum_{t=0}^{n-1} \beta^{t}\left(f\left(x_{t}\right)+g\left(u_{t}\right)\right)\right]
$$

and $\sum_{i=0}^{\infty} \beta^{i} E_{x}\left[f\left(x_{i}\right)\right]<\infty$ then it is uniformly in $n$ bounded by $m_{\beta}(x)$. Consequently there is a limit $w^{\beta}(x)$ of $w^{\beta, n}(x)$. It is also a convex and even function. Therefore $w^{\beta}(x)$ is also a continuous function and by Dini's theorem $w^{\beta, n}(x)$ converges to $w^{\beta}(x)$ uniformly on compact sets. Moreover the following equality is satisfied

$$
w^{\beta, n+1}(x)=f(x)+\inf _{u}\left[g(u)+\beta E_{x}\left[w^{\beta, n}\left(a x+b u+c W_{1}\right)\right]\right] .
$$


Let $G^{n}(x):=E_{x}\left[w^{\beta, n}\left(x+c W_{1}\right)\right]$. By Lemma $2.1 G^{n}$ is a strictly convex and even function. Then by Lemma 2.2 the infimum in (24) is attained for $u$ from the interval $\left[-\left|\frac{a x}{b}\right|,\left|\frac{a x}{b}\right|\right]$. Since $G^{n}(x) \leq m_{\beta}(x)$ and $G^{n}(x)$ converges to $G(x)=E_{x}\left[w\left(x+c W_{1}\right)\right]$, which is again convex and therefore continuous, by Dini's theorem $G^{n}$ converges to $G$ uniformly on compact sets. Therefore let $n \rightarrow \infty$ in (24), which leads to the Bellman equation 21.

5. Average cost per unit time problem. Now consider the minimization of the following long run average cost functional

$$
J_{x}^{a}(V)=\limsup _{T \rightarrow \infty} \frac{1}{T} E_{x}\left[\sum_{t=0}^{T-1}\left(f\left(x_{t}\right)+g\left(u_{t}\right)\right)\right]
$$

over strategies $V=\left(u_{0}, \ldots, u_{T-1}, \ldots\right)$. By a Tauberian Lemma (see Lemma 8.3.1 in [15]) it follows directly that

$$
\limsup _{\beta \rightarrow 1}(1-\beta) J_{x}^{\beta}(V) \leq J_{x}^{a}(V)
$$

Consequently

$$
\limsup _{\beta \rightarrow 1}(1-\beta) \inf _{V} J_{x}^{\beta}(V) \leq \inf _{V} J_{x}^{a}(V) .
$$

Let $\bar{w}^{\beta}(x)=w^{\beta}(x)-w^{\beta}(0)$. Since $w^{\beta}$ is convex and even, its minimum is at $x=0$ and therefore $\bar{w}^{\beta}(x) \geq 0$. Furthermore by 21 it is a solution to

$$
\bar{w}^{\beta}(x)+(1-\beta) w^{\beta}(0)=f(x)+\inf _{u}\left[g(u)+\beta E\left[\bar{w}^{\beta}\left(a x+b u+c W_{1}\right)\right]\right] .
$$

In what follows using a vanishing discount approach it is desired to let $\beta \rightarrow 1$ in (28). The following result is valid.

TheOrem 5.1. Assume that $E\left\{f\left(d+c W_{1}\right)\right\}<\infty$ and $E\left\{g\left(d+e W_{1}\right)\right\}<\infty$, for each $d, e \in R$ and $\liminf _{\beta \rightarrow 1}(1-\beta) m_{\beta}(0)<\infty$. Then there $i s$ a constant $\lambda$ and a convex and even function $w \geq 0$ that are solutions to the equation

$$
w(x)+\lambda=f(x)+\inf _{u}\left[g(u)+E\left[w\left(a x+b u+c W_{1}\right)\right]\right] .
$$

Proof. We are going to show that the family $\left\{\bar{w}^{\beta}(x), \beta \in(0,1)\right\}$ is locally bounded (i.e. bounded on any compact set) and is equicontinuous at each point. By assumption choose a subsequence $\beta_{n} \rightarrow 1$ such that $\left(1-\beta_{n}\right) m_{\beta_{n}}(0)$ is bounded. Then there is a further subsequence, for simplicity still denoted by $\beta_{n}$, such that $\left(1-\beta_{n}\right) w^{\beta_{n}}(0)$ converges to $\lambda$. By Ascoli-Arzela theorem (see Theorem 2.3 in [3]) choose a further subsequence (again denoted by $\beta_{n}$ ) such that $\bar{w}^{\beta_{n}}(x)$ converges uniformly in $x$ from compact subsets to a continuous function $w(x)$. Since the functions $\bar{w}^{\beta}$ are convex and even, the function $w$ is convex and even as well. Then we show in Step 3 that we are allowed let $\beta_{n} \rightarrow 1$ in (28, which completes the proof. The proof now consists of several steps:

Step 1. Local boundedness of $\left\{\bar{w}^{\beta}(x), \beta \in(0,1)\right\}$. The following inequalities are satisfied

$$
\begin{aligned}
0 \leq \bar{w}^{\beta}(x)=w^{\beta}(x)-w^{\beta}(0) \leq & f(x)+g\left(\frac{-a x}{b}\right)+\beta E\left[\bar{w}^{\beta}\left(c W_{1}\right)\right] \\
& -f(0)-g(0)-\beta E\left[\bar{w}^{\beta}\left(c W_{1}\right)\right]=\bar{f}(x)+\bar{g}\left(\frac{-a x}{b}\right)
\end{aligned}
$$

where $\bar{f}(x)=f(x)-f(0)$ and $\bar{g}(x)=g(x)-g(0)$. 
Step 2. Equicontinuity at each point of $\bar{w}^{\beta}$. Denote by $\hat{u}^{\beta}$ the selector minimizing right hand side of 21 . Then it follows that

$$
\begin{aligned}
\bar{w}^{\beta}(x)-\bar{w}^{\beta}(y) \leq & f(x)+g\left(\hat{u}^{\beta}(y)+\frac{y-x}{b}\right)+\beta E\left[\bar{w}^{\beta}\left(a y+b \hat{u}^{\beta}(y)+c W_{1}\right)\right] \\
& -f(y)-g\left(\hat{u}^{\beta}(y)\right)-\beta E\left[\bar{w}^{\beta}\left(a y+b \hat{u}^{\beta}(y)+c W_{1}\right)\right] \\
& =f(x)-f(y)+g\left(\hat{u}^{\beta}(y)+\frac{y-x}{b}\right)-g\left(\hat{u}^{\beta}(y)\right) .
\end{aligned}
$$

Since by Lemma $2.2\left|\hat{u}^{\beta}(y)\right| \leq\left|\frac{a y}{b}\right|$ equicontinuity at each point follows.

Step 3. Take the limit in (28) along a suitable subsequence. Note first that $\bar{w}^{\beta}$ is convex and even and therefore $\bar{w}^{\beta} \geq 0$. Moreover by Lemma 2.2 infimum in 28 is attained for $u$ between 0 and $\frac{-b x}{2 a}$, that does not depend on $\beta$ and by Step $1 \bar{w}^{\beta}\left(a x+b u+c W_{1}\right) \leq$ $\bar{f}\left(a x+b u+c W_{1}\right)+\bar{g}\left(-a \frac{a x+b u+c W_{1}}{b}\right)$ and both functions are integrable. Consequently

$$
E\left[\bar{w}^{\beta_{n}}\left(a x+b u+c W_{1}\right)\right] \rightarrow E\left[\bar{w}\left(a x+b u+c W_{1}\right)\right]
$$

uniformly in $u$ taking values between 0 and $\frac{-b x}{2 a}$, as $\beta_{n} \rightarrow 1$. Therefore let the subsequence $\beta_{n} \rightarrow 1$ in 28 from which 29 follows.

Corollary 5.2. For any control $V$ it follows that $\lambda \leq \inf _{V} J_{0}^{a}(V) \leq \inf _{V} J_{x}^{a}(V)$. For the control $\hat{V}$ consisting of controls for which equality in $(29)$ is satisfied, we have $\lambda=J_{x}^{a}(\hat{V})$.

Proof. The first inequality follows from the proof of Theorem 5.1 and inequality (26). Note now that for $V=\left(u_{0}, u_{1}, \ldots\right)$ and $V^{\prime}=\left(\frac{a x}{b}+u_{0}, u_{1}, \ldots\right)$ it follows that $x_{1}^{V}=$ $a x+b u_{0}+c W_{1}$ with $x_{0}=x$ under $V$ and $x_{1}^{V^{\prime}}=a x_{0}+b u_{0}+c W_{1}=x_{1}^{V}$ with $x_{0}=0$ under $V^{\prime}$. Therefore

$$
E_{x}\left[\sum_{t=0}^{T-1}\left(f\left(x_{t}^{V}\right)+g\left(u_{t}\right)\right)\right]=E_{0}\left[\sum_{t=1}^{T-1}\left(f\left(x_{t}^{V^{\prime}}\right)+g\left(u_{t}\right)\right)\right]+f(x)+g\left(u_{0}\right)
$$

and consequently $J_{x}^{a}(V)=J_{0}^{a}\left(V^{\prime}\right)$. Thus $\inf _{V} J_{x}^{a}(V) \geq \inf _{V} J_{0}^{a}(V) \geq \lambda$.

For the control $\hat{V}$

$$
w\left(x_{n}\right)+\lambda=f\left(x_{n}\right)+g\left(u_{n}\right)+E\left[w\left(x_{n+1}\right) \mid x_{0}, \ldots, x_{n}\right] .
$$

Therefore by summing first $T-1$ equations in (34) it follows that

$$
w(x)+T \lambda=E\left[\sum_{i=0}^{T-1}\left(f\left(x_{n}\right)+g\left(u_{n}\right)\right)+w\left(x_{T}\right)\right]
$$

and since $w \geq 0$ it follows that $\lambda \geq J_{x}^{a}(\hat{V})$ and by the first part of the proof there is the equality.

REMARK 5.3. Note that in the discounted problem and now also in the average cost per unit time problem it was important to know that optimal controls, which may depend in the discounted case on $n$ and in the average cost per unit time on $\beta$ in the problems approximating the studied problem, are from a given compact set (in our case an interval), which does not depend on these parameters. This allows one to insert the limit under the infimum sign. Such a property does not hold in general in the multidimensional control case. One can easily construct suitable counterexamples. 
6. Finite time horizon exponential functional. Now consider a discounted exponential functional

$$
J_{x}^{e T}(V)=\ln \left(E_{x}\left[\exp \left\{\sum_{t=0}^{T-1}\left(f_{t}\left(x_{t}\right)+g_{t}\left(u_{t}\right)\right)+G\left(x_{T}\right)\right\}\right]\right)
$$

where $f_{t}, g_{t}$ and $G$ are nonnegative, strictly convex and even for $t=0,1, \ldots, T-1$ and $\left(x_{t}\right)$ follows 19 . The following technical lemma is used subsequently.

LEMMA 6.1. If $F$ is strictly convex and $E\left[e^{F\left(x+c W_{1}\right)}\right]$ is well defined for each $x$, then

$$
E\left[e^{F\left(x+c W_{1}\right)}\right]=e^{\tilde{F}(x)}
$$

where $\tilde{F}$ is strictly convex.

Proof. Let $\tilde{F}(x)=\ln E\left[e^{F\left(x+c W_{1}\right)}\right]$. Then by using the strict convexity of $F$ and the Hölder inequality for $\lambda \in[0,1]$ the following inequality is satisfied:

$$
\begin{aligned}
E\left[e^{F\left(\lambda x+(1-\lambda) y+c W_{1}\right)}\right] & \leq E\left[e^{\lambda F\left(x+c W_{1}\right)+(1-\lambda) F\left(y+c W_{1}\right)}\right] \\
& =\left(E\left[e^{F\left(x+c W_{1}\right)}\right]\right)^{\lambda}\left(E\left[e^{F\left(y+c W_{1}\right)}\right]\right)^{1-\lambda}=e^{\lambda \tilde{F}(x)} e^{(1-\lambda) \tilde{F}(y)}
\end{aligned}
$$

with strict inequality for $\lambda \in(0,1)$, which completes the proof.

COROLlary 6.2. For strictly convex and even functions $F$ and $g$ and constants $a$ and $b$, assuming that $E\left[e^{F\left(x+c W_{1}\right)}\right]$ is well defined for each $x$ the function $H$ defined by

$$
H(x):=\inf _{u}\left[g(u)+\ln \left(E\left[e^{F\left(a x+b u+c W_{1}\right)}\right]\right)\right]
$$

is even and strictly convex. Furthermore the minimum in 39 is attained in the interval $\left[0, \frac{-a x}{b}\right]$ whenever $\frac{-a x}{b}>0$ and in the interval $\left[\frac{-a x}{b}, 0\right]$ whenever $\frac{-a x}{b}<0$, and at 0 when $a=0$ or $x=0$.

Proof. Note that $H(x)=\inf _{u}[g(u)+\tilde{F}(a x+b u)]$ is by Lemmas 6.1 and 2.2 strictly convex. Since $F$ and $g$ are even functions by Lemma 2.1 and 2.2 the function $H$ is also even and the minimum is attained in the intervals described above. Note that $H(0)=$ $g(0)+\ln \left(E^{\tilde{F}\left(c W_{1}\right)}\right)$, that is, for $x=0$ the optimal control is $u=0$.

Consider now the following system of Bellman equations

$$
\begin{aligned}
e^{w_{0}^{e}(x)} & =e^{G(x)} \\
e^{w_{n+1}^{e}(x)} & =\inf _{u} \exp \left(f_{T-(n+1)}(x)+g_{T-(n+1)}(u)\right) E\left[\exp \left(w_{n}^{e}\left(a x+b u+c W_{1}\right)\right)\right] \\
& \text { for } n=0,1, \ldots, T-2 \\
e^{w_{T}^{e}(x)} & =\inf _{u} e^{f_{0}(x)+g_{0}(u)} E\left[\exp \left(w_{T-1}^{e}\left(a x+b u+c W_{1}\right)\right)\right] .
\end{aligned}
$$

Optimal strategies are described in the following theorem.

THEOREM 6.3. Assume that for each $x$

$$
E_{x}\left[\exp \left(\sum_{t=0}^{T-1-k} f_{t}\left(x_{t}\right)+G\left(x_{T-k}\right)\right)\right]<\infty,
$$

for $k=0,1, \ldots, T-1$, where $x_{n}$ corresponds to the state process with control $u \equiv 0$ and the functions $f_{t}, g_{t}$ and $G$ are nonnegative, strictly convex and even. Then functions $w_{n}$ defined in 40 for $n=0,1, \ldots, T$ are well defined, strictly convex and even and 
$w_{T}(x)=\sup _{V} J_{x}^{e T}(V)$. Furthermore optimal strategies at time $n$, for $n=0,1, \ldots, T-1$, are of the form $u_{n}\left(x_{n}\right)$ where $u_{n}$ is a uniquely defined continuous selector in the equation for $w_{T-n}$.

Proof. With the assumption (41) the right hand sides of the equations 40 are well defined. Then use Lemma 6.1 and Corollary 6.2 to obtain that the family $w_{n}$ is strictly concave and even. The remaining part of the proof is rather standard (see e.g. [11]).

7. Discounted exponential functional. Now consider the following exponential functional for $\beta, \gamma \in(0,1)$

$$
J_{x}^{e, \beta}(V, \gamma)=\ln \left(E_{x}\left[\exp \left\{\sum_{t=0}^{\infty} \beta^{t} \gamma\left(f\left(x_{t}\right)+g\left(u_{t}\right)\right)\right\}\right]\right)
$$

with dynamics of $\left(x_{n}\right)$ of the form 19 . A solution of the following Bellman equation is sought:

$$
\begin{array}{r}
\exp \left(w^{e, \beta}(x, \gamma)\right)=\inf _{u}\left[\exp (\gamma(f(x)+g(u))) E\left\{\exp \left(w^{e, \beta}\left(a x+b u+c W_{1}, \gamma \beta\right)\right)\right\}\right] \\
=: \exp \left(T^{e, \beta} w^{e, \beta}(x, \gamma)\right)
\end{array}
$$

where $f$ and $g$ are nonnegative, strictly convex and even.

Assume that $f$ and $g$ are nonnegative and $g(0)=0$. Then

$$
T^{e, \beta} 0(x, \gamma)=\inf _{u} \ln \left(e^{\gamma(f(x)+g(u))}\right)=\gamma f(x)=: w_{0}^{e, \beta}(x, \gamma) \geq 0,
$$

and the mapping $x \mapsto T^{e, \beta} 0(x, \gamma)$ is convex. Therefore $\left(T^{e, \beta}\right)^{n} 0(x, \gamma):=w_{n}^{e, \beta}(x, \gamma)$ is an increasing sequence, and by Corollary 6.2 it consists of convex functions.

Furthermore there is equality

$$
\exp \left(w_{n+1}^{e, \beta}(x, \gamma)\right)=\inf _{u}\left[e^{\gamma(f(x)+g(u))} E\left\{\exp \left(w_{n}^{e, \beta}\left(a x+b u+c W_{1}, \gamma \beta\right)\right)\right\}\right] .
$$

THEOREM 7.1. Assume that $\tilde{m}_{\beta}(x, \gamma):=E\left[\exp \left(\sum_{i=0}^{\infty} \gamma \beta^{i} f\left(x_{i}\right)\right)\right]<\infty$ where $\left(x_{i}\right)$ corresponds to the state process with the control $u_{n} \equiv 0$, for $n=0,1, \ldots$ Then there is a solution $w^{e, \beta}$ to the Bellman equation (43) which is convex in $x$, even, convex in $\gamma$ and $w^{e, \beta}(x, \gamma)=\inf _{V} J_{x}^{e, \beta}(V)$.

Proof. Note that $w_{n}^{e, \beta}(x, \gamma)$ is an optimal value of the functional

$$
J_{x}^{e, \beta, n}(x, \gamma, V)=\ln \left(E_{x}\left[\exp \left(\gamma \sum_{t=0}^{n-1} \beta^{t}\left(f\left(x_{t}\right)+g\left(u_{t}\right)\right)\right)\right]\right)
$$

and is uniformly bounded by $\ln \left(\tilde{m}_{\beta}(x, \gamma)\right)$. Consequently there is a limit $w^{e, \beta}(x, \gamma)$ of $w_{n}^{e, \beta}(x, \gamma)$. Since $x \mapsto w_{n}^{e, \beta}(x, \gamma)$ is strictly convex, the function $x \mapsto w^{e, \beta}(x, \gamma)$ is convex as well. Therefore $x \mapsto w^{e, \beta}(x, \gamma)$ is continuous and by Dini's theorem for each $\gamma$ function $w_{n}^{e, \beta}(x, \gamma)$ converges uniformly on compact sets to $w^{e, \beta}(x, \gamma)$. Moreover by Corollary 6.2 the functions $w_{n}^{e, \beta}$ are even and therefore $w^{e, \beta}$ is also even. Let $G^{n}(x, \gamma):=$ $\ln \left(E\left\{\exp \left(w_{n}^{e, \beta}\left(a x+b u+c W_{1}, \gamma\right)\right)\right\}\right)$. By Corollary 6.2 the function $x \mapsto G^{n}(x, \gamma)$ is strictly convex and even. Furthermore $G^{n}(x, \gamma) \leq \tilde{m}_{\beta}(x, \gamma)$. Sequence $G^{n}(x, \gamma)$ is increasing and converges to $G(x, \gamma)$ and $x \mapsto G(x, \gamma)$ is convex and therefore continuous. Consequently $G^{n}(x, \gamma)$ converges uniformly to $G(x, \gamma)$ for fixed $\gamma$. By Corollary 6.2 the 
infimum in (44) is attained for $u$ from the interval $\left[-\left|\frac{a x}{b}\right|,\left|\frac{a x}{b}\right|\right]$. By letting $n$ to $\infty$ in (44) it follows that $w^{e, \beta}$ is a solution to (43). Moreover note that $w_{n}^{e, \beta}(x, \gamma)$ is also convex in $\gamma$ because $J_{x}^{e, \beta, n}(x, \gamma, V)$ is convex for fixed $x$ and each $V$ (which follows in a similar way as the proof of Lemma 6.1. Therefore $w^{e, \beta}$ is convex in $\gamma$. The fact that $w^{e, \beta}$ is a value function follows in a standard way.

8. Long run exponential functional. Now consider the minimization of

$$
J_{x}^{e l, \gamma}(V)=\limsup _{T \rightarrow \infty} \frac{1}{T} \ln \left(E_{x}\left[\exp \left\{\gamma \sum_{t=0}^{T-1}\left(f\left(x_{t}\right)+g\left(u_{t}\right)\right)\right\}\right]\right) .
$$

This kind of functional is in a sense motivated by risk sensitive control problems and the method is based on a vanishing discount approach which follows some ideas of [14]. Now consider solving the following Bellman equation, that is, to find a constant $\lambda^{e}$ and a function $w$ such that

$$
\exp \left(v(x, \gamma)-\lambda^{e}(\gamma)\right)=\inf _{u}\left[e^{\gamma(f(x)+g(u))} E\left\{\exp \left(v\left(a x+b u+c W_{1}, \gamma\right)\right)\right\}\right] .
$$

From equation (43) by letting $\bar{w}^{e, \beta}(x, \gamma):=\bar{w}^{e, \beta}(x, \gamma)-w^{e, \beta}(0, \gamma)$ it follows that

$$
\begin{aligned}
\exp \left(\bar{w}^{e, \beta}(x, \gamma)+w^{e, \beta}(0, \gamma)\right. & \left.-w^{e, \beta}(0, \gamma \beta)\right) \\
= & \inf _{u}\left[e^{\gamma(f(x)+g(u))} E\left\{\exp \left(\bar{w}^{e, \beta}\left(a x+b u+c W_{1}, \gamma \beta\right)\right)\right\}\right] .
\end{aligned}
$$

Furthermore note that $\bar{w}^{e, \beta}(x, \gamma) \geq 0$ since each function $\bar{w}^{e, \beta}(x, \gamma)$ attains its minimum at 0 . For $x=0$ in 43 the infimum is attained by choosing $u=0$ so that

$$
\begin{aligned}
\exp \left(\bar{w}^{e, \beta}(x, \gamma)\right) \leq \frac{\exp \left(\gamma\left(f(x)+g\left(\frac{-a x}{b}\right)\right)\right) E\left\{\exp \left(w^{e, \beta}\left(c W_{1}, \gamma \beta\right)\right)\right\}}{\exp (\gamma(f(0)+g(0))) E\left\{\exp \left(w^{e, \beta}\left(c W_{1}, \gamma \beta\right)\right)\right\}} & =\exp \left(\gamma\left(\bar{f}(x)+\bar{g}\left(\frac{-a x}{b}\right)\right)\right)
\end{aligned}
$$

where $\bar{f}(x)=f(x)-f(0)$ and $\bar{g}(x)=g(x)-g(0)$. Let $u_{x}$ be an optimal control for $x$. Then

$$
\begin{array}{r}
\exp \left(\bar{w}^{e, \beta}(x, \gamma)\right) \geq \frac{\exp \left(\gamma\left(f(x)+g\left(u_{x}\right)\right)\right) E\left\{\exp \left(w^{e, \beta}\left(a x+b u_{x}+c W_{1}, \gamma \beta\right)\right)\right\}}{\exp \left(\gamma\left(f(0)+g\left(\frac{a x}{b}+u_{x}\right)\right)\right) E\left\{\exp \left(w^{e, \beta}\left(a x+b u_{x}+c W_{1}, \gamma \beta\right)\right)\right\}} \\
=\exp \left(\gamma\left(\bar{f}(x)+g\left(u_{x}\right)-g\left(\frac{a x}{b}+u_{x}\right)\right)\right)
\end{array}
$$

and

$$
\begin{array}{r}
\exp \left(w^{e, \beta}(x, \gamma)-w^{e, \beta}(y, \gamma)\right) \\
\leq \frac{\exp \left(\gamma\left(f(x)+g\left(u_{y}+\frac{y-x}{b}\right)\right)\right) E\left\{\exp \left(w^{e, \beta}\left(a y+b u_{y}+c W_{1}, \gamma \beta\right)\right)\right\}}{\exp \left(\gamma\left(f(y)+g\left(u_{y}\right)\right)\right) E\left\{\exp \left(w^{e, \beta}\left(a y+b u_{y}+c W_{1}, \gamma \beta\right)\right)\right\}} \\
\quad=\exp \left(\gamma\left(f(x)-f(y)+g\left(u_{y}+\frac{y-x}{b}\right)-g\left(u_{y}\right)\right)\right) .
\end{array}
$$

THEOREM 8.1. Assume that $\tilde{m}_{\beta}(x, \gamma)<\infty$ for each $x$ and

$$
E\left[\exp \left(\gamma \beta\left(f\left(c W_{1}\right)+g\left(\frac{-a c W_{1}}{b}\right)\right)\right)\right]<\infty
$$


for all $\beta, \gamma \in(0,1)$. Then there is a constant $\lambda^{e}(\gamma)$ and nonnegative continuous even convex functions $v^{e}$, for $n=0,1, \ldots$, such that

$$
\exp \left(v^{e}(x, \gamma)+\lambda^{e}(\gamma)\right)=\inf _{u}\left[e^{\gamma(f(x)+g(u))} E\left\{\exp \left(v^{e}\left(a x+b u+c W_{1}, \gamma\right)\right)\right\}\right] .
$$

Furthermore functions $v^{e}$ have the following uniform bounds

$$
\inf _{|u| \in\left[0,\left|\frac{b}{a}\right|\right]} \exp \left(\gamma\left(\bar{f}(x)+g(u)-g\left(\frac{a x}{b}+u\right)\right)\right) \leq e^{v^{e}(x, \gamma)} \leq \exp \left(\gamma\left(\bar{f}(x)+\bar{g}\left(\frac{-a x}{b}\right)\right)\right)
$$

and there is the inequality

$$
\lambda^{e}(\gamma) \geq J_{x}^{e l, \gamma}((\hat{u}))
$$

for control functions $\hat{u}$ which is a selector in the equation (53). Furthermore if for a given control $V=\left(u_{0}, u_{1}, \ldots\right)$ we define a sequence of finite horizon controls

$$
V_{T}=\left(u_{0}, u_{1}, \ldots, u_{T-2}, u_{T-1}=-a x_{T-1} / b\right)
$$

then for such a sequence of controls we have the inequality

$$
\limsup _{T \rightarrow \infty} \frac{1}{T} \ln \left(E_{x}\left[\exp \left\{\gamma \sum_{t=0}^{T-1}\left(f\left(x_{t}^{V_{T}}\right)+g\left(u_{t}\right)\right)\right\}\right]\right) \geq \lambda^{e}(\gamma) .
$$

Proof. Since by Corollary 6.2 an optimal control $u_{x}$ is between 0 and $\frac{-a}{2 b}$ it follows that for each $\beta$ and $\gamma \in(0,1)$ functions $\bar{w}^{e, \beta}(\cdot, \gamma)$ are locally bounded equicontinuous at each point (uniformly in $\beta$ and $\gamma$ ). Therefore by Theorem 2.3 in [3] for each $\gamma$ there is a convergent subsequence $\beta_{n} \rightarrow 1$ such that for each $m=0,1, \ldots$ it follows that $\bar{w}^{e, \beta_{n}}\left(x, \gamma \beta_{n}^{m}\right) \rightarrow w_{m}^{e}(x, \gamma)$ as $n \rightarrow \infty$ uniformly in $x$ from compact sets. Furthermore $w_{m}^{e}(\cdot, \gamma)$ are convex and even functions because such are the functions $\bar{w}^{e}\left(x, \gamma \beta^{m}\right)$. It is clear that $w^{e, \beta}\left(0, \gamma \beta^{m}\right)-w^{e, \beta}\left(0, \gamma \beta^{m+1}\right) \geq 0$. On the other hand using 49 we obtain for each $\gamma \in(0,1)$, and $m=0,1, \ldots$

$$
\begin{aligned}
\exp \left(w^{e}\left(0, \gamma \beta^{m}\right)\right) & =e^{\gamma(f(0)+g(0))} E\left\{\exp \left(w^{e, \beta}\left(c W_{1}, \gamma \beta^{m+1}\right)\right)\right\} \\
= & e^{\gamma(f(0)+g(0))} E\left\{\exp \left(\bar{w}^{e, \beta}\left(c W_{1}, \gamma \beta^{m+1}\right)\right)\right\} \exp \left(w^{e, \beta}\left(0, \gamma \beta^{m+1}\right)\right) .
\end{aligned}
$$

Therefore $\lambda_{m}^{e, \beta_{n}}(\gamma):=w^{e, \beta_{n}}\left(0, \gamma \beta_{n}^{m}\right)-w^{e, \beta_{n}}\left(0, \gamma \beta_{n}^{m+1}\right)$ converges to $\lambda_{m}^{e}(\gamma)$ as $n \rightarrow \infty$. Moreover by convexity with respect to $\gamma$ it follows for $m=0,1, \ldots$ that

$$
0 \leq \frac{w^{e, \beta}\left(x, \gamma \beta^{m}\right)-w^{e, \beta}\left(x, \gamma \beta^{m+1}\right)}{\gamma \beta^{m}(1-\beta)} \leq \frac{w^{e, \beta}\left(x, \gamma \beta^{m+1}\right)-w^{e, \beta}\left(x, \gamma \beta^{m+2}\right)}{\gamma \beta^{m+1}(1-\beta)}
$$

and therefore in particular

$$
\beta_{n} \lambda_{m}^{e, \beta_{n}}(\gamma) \leq \lambda_{m+1}^{e, \beta_{n}}(\gamma)
$$

Consequently $\lambda_{m}^{e}(\gamma)$ is nondecreasing in $m$. Since in 48 the controls $u$ can be restricted between 0 and $\frac{-a x}{b}$, letting $\beta^{n} \rightarrow 1$ in 48 we obtain for $m=0,1, \ldots$

$$
\exp \left(w_{m}^{e}(x, \gamma)+\lambda_{m}^{e}(\gamma)\right)=\inf _{u}\left[e^{\gamma(f(x)+g(u))} E\left\{\exp \left(w_{m+1}^{e}\left(a x+b u+c W_{1}, \gamma\right)\right)\right\}\right] .
$$

Note that by (49)-51) for functions $w_{m}^{e}(x, \gamma)$ it follows that

$$
\inf _{|u| \in\left[0,\left|\frac{b}{a}\right|\right]} \exp \left(\gamma\left(\bar{f}(x)+g(u)-g\left(\frac{a x}{b}+u\right)\right)\right) \leq e^{w_{m}^{e}(x, \gamma)} \leq \exp \left(\gamma\left(\bar{f}(x)+\bar{g}\left(\frac{-a x}{b}\right)\right)\right)
$$

and $0 \leq \lambda_{m}^{e}(\gamma) \leq K(\gamma)$, where $K(\gamma)$ is a constant depending on $\gamma$. Now

$$
\bar{w}^{e, \beta_{n}}\left(x, \gamma \beta_{n}^{m}\right)-\bar{w}^{e, \beta_{n}}\left(x, \gamma \beta_{n}^{m+1}\right)=-\lambda_{m}^{e, \beta_{n}}(\gamma)+w^{e, \beta_{n}}\left(x, \gamma \beta_{n}^{m}\right)-w^{e, \beta_{n}}\left(x, \gamma \beta_{n}^{m+1}\right)
$$


and since from 58

$$
\beta_{n}\left(w^{e, \beta_{n}}\left(x, \gamma \beta_{n}^{m}\right)-w^{e, \beta_{n}}\left(x, \gamma \beta_{n}^{m+1}\right)\right) \leq w^{e, \beta_{n}}\left(x, \gamma \beta_{n}^{m+1}\right)-w^{e, \beta_{n}}\left(x, \gamma \beta_{n}^{m+2}\right)
$$

by letting $n \rightarrow \infty$ it follows that

$$
w_{m}^{e}(x, \gamma)-w_{m+1}^{e}(x, \gamma)=-\lambda_{m}^{e}(\gamma)+z_{m}(x, \gamma),
$$

where $z_{m}$ is a nondecreasing sequence of functions. Clearly $\lambda_{m}^{e}(\gamma)$ converges to $\lambda^{e}(\gamma)$ and by (61) also $z_{m}(x, \gamma)$ converges to $z(x, \gamma)$. Therefore

$$
w_{m+1}^{e}(x, \gamma)=w_{m}^{e}(x, \gamma)+\lambda_{m}^{e}(\gamma)-z_{m}(x, \gamma)
$$

and if $\lambda^{e}(\gamma)>z(x, \gamma)$ letting $m \rightarrow \infty$ it follows that the sequence $w_{m+1}^{e}(x, \gamma)$ is unbounded, which contradicts 61, while if $\lambda^{e}(\gamma)<z(x, \gamma)$ it follows that $w_{m}^{e}(x, \gamma)$ is unbounded which again contradicts (61). Consequently $\lambda^{e}(\gamma)=z(x, \gamma)$ and

$$
\lim _{m \rightarrow \infty}\left(w_{m+1}^{e}(x, \gamma)-w_{m}^{e}(x, \gamma)\right)=0 .
$$

By (61) and Theorem 2.3 in 3] there is a subsequence $m_{k} \rightarrow \infty$ as well as a sequence of functions $v_{i}^{e}$ such that $w_{m_{k}+i}^{e}(x, \gamma) \rightarrow v_{i}^{e}(x, \gamma)$ for $i=0,1 \ldots$, uniformly on compact subsets. Using $\lim _{m \rightarrow \infty}\left(w_{m+1}^{e}(x, \gamma)-w_{m}^{e}(x, \gamma)\right)=0$ we find that the functions $v_{i}^{e}(x, \gamma)$ do not depend on $i$ and so this is denoted as $v^{e}(x, \gamma)$. Clearly $x \mapsto v^{e}(x, \gamma)$ is convex and even (since such is $x \mapsto w_{m}^{e}(x, \gamma)$ ). If $m_{k} \rightarrow \infty$ in equation (60), (53) follows. Furthermore it can be shown that for any control $\left(\hat{u}_{n}\right)$ which consists of the selectors in equation (53)

$$
\begin{array}{r}
e^{v_{n+1}^{e}(x, \gamma)+(m+1) \lambda^{e}(\gamma)}=\left[E_{x}\left\{\exp \left(\gamma \sum_{i=0}^{m}\left(f\left(X_{i}\right)+g\left(\hat{u}_{i}\right)\right)\right) \exp \left(v_{n-m}^{e}\left(X_{m+1}, \gamma\right)\right)\right\}\right] \\
\geq\left[E_{x}\left\{\exp \left(\gamma \sum_{i=0}^{m}\left(f\left(X_{i}\right)+g\left(\hat{u}_{i}\right)\right)\right)\right\}\right],
\end{array}
$$

since $v_{n-m}^{e} \geq 0$, so by taking the logarithm of both sides of 66 and dividing by $m+1$ (55) follows. Note now that from equation (53) in a similar way as in the finite horizon case there is the equality

$$
\exp \left(v_{n+1}^{e}(x, \gamma)+n \lambda^{e}(\gamma)\right)=\inf _{u_{n}} E_{x}\left[\exp \left\{\gamma \sum_{t=0}^{n}\left(f\left(x_{t}\right)+g\left(u_{t}\right)\right)+v^{e}\left(x_{n+1}, \gamma\right)\right\}\right]
$$

where $x_{n}$ corresponds to the state process corresponding to control sequence $\left(u_{n}\right)$. Consequently for any control $\left(u_{n}\right)$ there is the inequality

$$
E_{x}\left[\exp \left\{\gamma \sum_{t=0}^{n}\left(f\left(x_{t}\right)+g\left(u_{t}\right)\right)+v^{e}\left(x_{n+1}, \gamma\right)\right\}\right] \geq \exp \left(v_{n+1}^{e}(x, \gamma)+n \lambda^{e}(\gamma)\right) \geq e^{n \lambda^{e}(\gamma)}
$$

By (54) it follows that $v^{e}\left(x_{n+1}, \gamma\right) \leq \gamma\left(\bar{f}(x)+\bar{g}\left(\frac{-a x}{b}\right)\right)$ and therefore for $u_{n+1}=\frac{-a x_{n+1}}{b}$ we have the inequality

$$
E_{x}\left[\exp \left\{\gamma \sum_{t=0}^{n+1}\left(f\left(x_{t}^{V_{n+2}}\right)+g\left(u_{t}\right)\right)-f(0)-g(0)\right\}\right] \geq e^{n \lambda^{e}(\gamma)}
$$

from which 56 follows. 
9. Long run exponential functional-finite horizon approach. Now consider a finite horizon exponential Bellman equation. Let $\bar{w}_{n}^{e}(x)=w_{n}^{e}(x)-w_{n}^{e}(0)$. Then from (40) it follows that

$$
\exp \left(\bar{w}_{n+1}^{e}(x)+\left(w_{n+1}^{e}(0)-w_{n}^{e}(0)\right)\right)=\inf _{u} e^{f(x)+g(u)} E\left[\exp \left(\bar{w}_{n}^{e}\left(a x+b u+c W_{1}\right)\right)\right]
$$

A few estimates are needed now:

$$
\exp \left(\bar{w}_{n+1}^{e}(x)\right) \leq \frac{\exp \left(f(x)+g\left(\frac{-a x}{b}\right)\right) E\left\{\exp \left(w_{n}^{e}\left(c W_{1}\right)\right)\right\}}{e^{(f(0)+g(0))} E\left\{\exp \left(w_{n}^{e}\left(c W_{1}\right)\right)\right\}}=\exp \left(\bar{f}(x)+\bar{g}\left(\frac{-a x}{b}\right)\right)
$$

and for $n \geq 2$

$$
\begin{aligned}
& 1 \leq \exp \left(w_{n+1}^{e}(0)-w_{n}^{e}(0)\right) \leq e^{f(0)+g(0)} E\left\{\exp \left(\bar{w}_{n}^{e}\left(c W_{1}\right)\right)\right\} \\
& \quad \leq e^{f(0)+g(0)} E\left\{\exp \left(\bar{f}\left(c W_{1}\right)+\bar{g}\left(\frac{-a c W_{1}}{b}\right)\right)\right\} .
\end{aligned}
$$

Furthermore denoting by $\hat{u}_{n}$ the selector in 70 we obtain

$$
\begin{aligned}
& \exp \left(w_{n+1}^{e}(x)-w_{n+1}^{e}(y)\right) \\
& \leq \frac{\exp \left(f(x)+g\left(-\frac{a(x-y)-b \hat{u}_{n}(y)}{b}\right)\right) E\left\{\exp \left(w_{n}^{e}\left(a y+b \hat{u}_{n}(y)+c W_{1}\right)\right)\right\}}{\exp \left(f(y)+g\left(b \hat{u}_{n}(y)\right)\right) E\left\{\exp \left(w_{n}^{e}\left(a y+b \hat{u}_{n}(y)+c W_{1}\right)\right)\right\}} \\
& \quad=\exp \left(f(x)-f(y)+g\left(-\frac{a(x-y)-b \hat{u}_{n}(y)}{b}\right)-g(u(y))\right) .
\end{aligned}
$$

THEOREM 9.1. Assume that for each $x$

$$
E_{x}\left[\exp \left(\sum_{t=0}^{T-1-k} f\left(x_{t}\right)\right)\right]<\infty
$$

for $k=0,1, \ldots, T-1$, where $x_{n}$ corresponds to the state process with control $u \equiv 0$,

$$
E\left[\exp \left(f\left(c W_{1}\right)+g\left(\frac{-a c W_{1}}{b}\right)\right)\right]<\infty .
$$

and functions $f, g$ are nonnegative, strictly convex and even. Then there is a pair: a constant $\lambda^{e}$ and a sequence of functions $v_{n}^{e}$ that are solutions to the Bellman equations

$$
\exp \left(v_{n+1}^{e}(x, \gamma)+\lambda^{e}(\gamma)\right)=\inf _{u}\left[e^{\gamma(f(x)+g(u))} E\left\{\exp \left(v_{n}^{e}\left(a x+b u+c W_{1}, \gamma\right)\right)\right\}\right] .
$$

Furthermore the functions $v_{n}^{e}$ are convex and even and

$$
v_{n}^{e}(x) \leq \bar{f}(x)+\bar{g}\left(\frac{-a x}{b}\right) .
$$

Proof. With the assumption (74) the finite horizon exponential Bellman equations (40) have solutions $w_{n}^{e}$ which by Theorem 6.3 are strictly convex and even. Using (71) and (73) it follows that the family $\left\{\bar{w}_{n}^{e}, n=1,2, \ldots\right\}$ is locally bounded and uniformly continuous at each point. From $(50)$ one can choose a subsequence $n_{k}$ such that

$$
\exp \left(w_{n_{k}+i+1}^{e}(0)-w_{n_{k}+i}^{e}(0)\right) \rightarrow \lambda_{i}^{e}
$$

as $k \rightarrow \infty$ for $i=0,1,2, \ldots$ By 72 it follows that $\lambda^{e} \geq 0$. By Ascoli-Arzela arguments (see [3]) there is a further subsequence, which for simplicity is still denoted by $n_{k}$ such that $\bar{w}_{n_{k}+i}^{e}(x) \rightarrow \bar{v}_{i}^{e}(x)$ for $i=0,1, \ldots$ and the functions $\bar{v}_{i}^{e}$ are convex and even. Therefore by letting $k \rightarrow \infty$ in $\left(70\right.$, using that the infimum is attained between 0 and $\frac{-a x}{b}$ it follows that

$$
\exp \left(\bar{v}_{n+1}^{e}(x, \gamma)+\lambda_{n}^{e}(\gamma)\right)=\inf _{u}\left[e^{\gamma(f(x)+g(u))} E\left\{\exp \left(\bar{v}_{n}^{e}\left(a x+b u+c W_{1}, \gamma\right)\right)\right\}\right]
$$


Choosing a further subsequence $n_{k}$ we deduce that the $\operatorname{limits}_{k \rightarrow \infty} \lim _{n_{k}}^{e}(\gamma) \rightarrow \lambda^{e}(\gamma)$ and $\bar{v}_{n_{k}+i}^{e}(x) \rightarrow v_{i}^{e}(x)$ exist uniformly on compact subsets as $k \rightarrow \infty$, and therefore (76) is satisfied. By (71) we immediately obtain (77).

REMARK 9.2. Using finite horizon approximation we obtain weaker version of the Bellman equation (53). Instead of one function $v^{e}$ we have a sequence of convex and even functions $v_{i}^{e}$. We have however a uniform bound (77) for these functions. There are no reason to expect that $v_{i}^{e}$ do not depend on $i$ as we managed to show in the case when we were using discounted approximation (see the end of the proof of Theorem 8.1.

Acknowledgments. Research of T. Duncan and B. Pasik Duncan was supported by US NSF grant DMS 1411412 and AFOSR grant FA9550-17-1-0073. Research of $\mathrm{E}$. Stettner was supported by National Science Center by grant UMO-2016/23/B/ST1/00479.

\section{References}

[1] D. Bertsekas, Linear convex stochastic control problems over an infinite horizon, IEEE Trans. Automatic Control AC 18 (1973), 314-315.

[2] C. Bes, S. Sethi, Solution of a class of stochastic linear-convex control problems using deterministic equivalents, J. Optim. Theory Appl. 62 (1989), 17-27.

[3] R. F. Brown, A Topological Introduction to Nonlinear Analysis, Springer, Cham, 2014.

[4] A. Cadenillas, I. Karatzas, The stochastic maximum principle for linear convex control with random coefficients, SIAM J. Control Optim. 33 (1995), 590-624.

[5] T. Duncan, Stochastic linear-quadratic control revisited, in: Stochastic Processes, Finance and Control, Adv. Stat. Probab. Actuar. Sci. 1, World Sci., Hackensack, NJ, 2012, 451-463.

[6] T. Duncan, Linear-exponential-quadratic Gaussian control, IEEE Trans. Automat. Control 58 (2013), 2910-2911.

[7] T. Duncan, B. Pasik-Duncan, Linear-quadratic fractional Gaussian control, SIAM J. Control Optim. 51 (2013), 4504-4519.

[8] T. Duncan, B. Pasik-Duncan, Discrete time linear quadratic control with arbitrary correlated noise, IEEE Trans. Automat. Control 58 (2013), 1290-1293.

[9] I. V. Evstigneev, Measurable selection and dynamic programming, Math. Oper. Res. 1 (1976), 267-272.

[10] C. Fan, J. L. Speyer, C. R. Jaensch, Centralized and decentralized solutions of the linearexponential-Gaussian problem, IEEE Trans. Automat. Control 39 (1994), 1986-2003.

[11] O. Hernández-Lerma, J. B. Lasserre, Discrete-Time Markov Control Processes. Basic Optimality Criteria, Appl. Math. (N.Y.) 30, Springer, New York, 1996.

[12] O. Kallenberg, Foundations of Modern Probability, second ed., Probab. Appl. (N.Y.), Springer, New York, 2002.

[13] S. Sethi, M. Taksar, Deterministic equivalent for a continuous-time linear-convex stochastic control problem, J. Optim. Theory Appl. 64 (1990), 169-181.

[14] Ł. Stettner, Asymptotics of HARA utility from terminal wealth under proportional transaction costs with decision lag or execution delay and obligatory diversification, in: Advanced Mathematical Methods for Finance, Springer, Heidelberg, 2011, 509-536.

[15] J. Zabczyk, Chance and Decision. Stochastic Control in Discrete Time, Sc. Norm. Super. di Pisa Quaderni, Scuola Normale Superiore, Pisa, 1996. 